\title{
Tratamento da degeneração macular relacionada à idade com neovascularização de coróide extrafoveal. Análise de uma série de casos e revisão de literatura ${ }^{1}$
}

\author{
Treatment of nonsubfoveal choroidal neovascularization \\ in age-related macular degeneration. Analysis \\ of a cases series and revision paper
}

Miguel Hage Amaro', Flávia Araújo Hage Amaro², Aaron Brock Roller ${ }^{3}$, Cesar Tavares Motta ${ }^{4}$, Mario Martins dos Santos Motta ${ }^{5}$

\section{ResUMO}

A degeneração macular relacionada à idade (DMRI) é uma doença degenerativa da área central da retina freqüentemente associada à perda visual central, em pessoas acima de 55 anos de ambos os sexos, sendo a mais importante causa de cegueira irreversível em adultos nos países desenvolvidos e a terceira causa de cegueira no mundo. Objetivo: Avaliar a eficácia da injeção intravítrea de Ranibizumab (anti-VEGF) no tratamento da neovascularização de coróide não subfoveal, podendo ser extrafoveal ou justafoveal, causada pela degeneração macular relacionada à idade exsudativa e revisão da literatura. Métodos: Foi realizado um estudo descritivo e transversal com avaliação de quinze pacientes com neovascularização de coróide extrafoveal ou justafoveal causada por degeneração macular relacionada à idade. Os pacientes foram tratados com uma injeção intravítrea, por mês, no mínimo por três meses consecutivos, de Ranibizumab na dose de 0,5mg, no olho com neovascularização de coróide. Os mesmos foram examinados com um mês de intervalo entre as aplicações das injeções e acompanhados com exames complementares. Resultados: Os resultados da análise dos quinze pacientes que foram incluídos nesta série de casos mostraram que onze $(73,3 \%)$ pacientes apresentavam neovascularização de coróide extrafoveal e quatro $(26,6 \%)$ justafoveal. Oito $(53,3 \%)$ apresentavam a forma oculta e sete $(46,6 \%)$ tinham lesões clássicas. A média de injeções realizadas foi de 3,67, sendo o menor número de injeções 3 e o maior 6 injeções. Oito (53,3\%) pacientes apresentaram resolução do quadro exsudativo neovascular com o máximo de três injeções e apenas um $(6,67 \%)$ paciente necessitou de seis injeções para resolução do seu quadro. A acuidade visual máxima alcançada foi de 20/30, e ocorreu em cinco (33,3\%) pacientes. A média de acuidade visual no momento do diagnóstico foi de 0,44logMAR e a média final de 0,27logMAR Na avaliação final dos pacientes após os seis meses de estudo, a média de linha de visão ganha foi de 1,87 linha de acuidade visual pela tabela de Snellen, sendo que todos os 15 pacientes ganharam uma ou mais linhas de visão. Conclusão: No tratamento dos pacientes com degeneração macular relacionada à idade e neovascularização de coróide extrafoveal a injeção de Ranibizumab mostrou-se eficaz, podendo ser a opção como indicação inicial de tratamento nestes casos.

Descritores: Inibidores da angiogênese/uso terapêutico; Anticorpos monoclonais/uso terapêutico; Degeneração macular /quimioterapia; Neovascularização coroidal/quimioterapia; Fóvea central/efeito de drogas

'Doutor em Oftalmologia pela Universidade Federal de São Paulo - UNIFESP - São Paulo (SP), Brasil;

${ }^{2}$ Acadêmica do Curso de Medicina da Universidade do Estado do Pará - UEPA - Belém (PA), Brasil;

${ }^{3}$ Departament of Ophthalmology, Vitreous Retina Service, University of lowa - USA;

${ }^{4}$ Médico Residente, Universidade do Rio de Janeiro - UNIRIO, Rio de Janeiro (RJ), Brasil;

${ }^{5}$ Professor, Universidade do Rio de Janeiro - UNIRIO, Rio de Janeiro (RJ), Brasil.

Trabalho de realizado com dados do Ophthalmology Department, University of lowa - USA

The autors received no public or private financial support

Recebido para publicação em: 25/1/2010 - Aceito para publicação em 3/6/2011 


\begin{abstract}
Age-related macular degeneration is a major cause of central vision loss and is the leading cause of blindness for people aged over 60 years. Purpose: To investigate the efficacy of intravitreal injection of Ranibizumab (anti-VEGF) in the treatment of choroidal neovascularization nonsubfoveal, extra-foveal or juxta-foveal, caused by age-related macular degeneration and revision paper. Methods: The study design was descriptive and transverse.15 patients with nonsubfoveal choroidal neovascularization caused by age-related macular degeneration, were treated with intravitreal injection of Ranibzumab per month, at least for three consecutive months. Patients were examined at one month interval between the injections and evaluated using visual acuity testing with Snellen charts, fluorescein angiography, and optical coherence tomography scans. Results: There were 11extra-foveal lesions $(73.3 \%)$ and 4 juxta-foveal lesions (26.6\%). Seven lesions were predominantly classic (46.6\%) and eight lesions were occult (53,3\%). The mean number of injections performed was 3.67 and the lowest number of injections 3 and 6 larger injections. Eight $(53.3 \%)$ patients had complete resolution of the exudative neovascular condition with a maximum of three injections and only one $(6.67 \%)$ patient required six injections to resolution his condition. The best visual acuity achieved was 20/30, and occurred in five $(33.3 \%)$ patients. The mean visual acuity at diagnosis was $0.44 \log M A R$ and $0.27 \log M A R$ final average. In the final evaluation of patients after six months of study, the average line of sight gains were 1.87 line of visual acuity by Snellen chart. All 15 patients gained one or more lines of vision. Conclusion: In the treatment of patients with nonsubfoveal choroidal neovascularization in age-related macular degeneration, injection of Ranibizumab was effective and could be an option as an indication of initial treatment in these cases.

Keywords: Angiogenesis inhibitors/therapeutic use; Antibodies, monoclonal; Macular degeneration/drug therapy; Choroidal neovascularization/drug therapy; Fovea centralis/drug effects
\end{abstract}

\section{INTRODUÇÃO}

A Degeneração macular relacionada à idade (DMRI) é uma das mais importantes causas de diminuição da acuidade visual em pacientes entre 55 anos e mais velhos ${ }^{(1)}$. Estima-se que, em 2011, haverá 239.000 pessoas no Reino Unido com diminuição da visão causada pela DMRI. Nos Estados Unidos, mais de 7 milhões de pessoas têm drusas de 125 mícrons ou maiores, estando, portanto, em risco substancial de desenvolver DMRI ${ }^{(2)}$.

Existem dois tipos de degeneração macular: seca e exsudativa (também chamada de neovascularizada) ${ }^{(3,4)}$.

O tipo seca, em sua forma inicial, muitas vezes evolui para seu estágio final caracterizado, como degeneração macular relacionada à idade geográfica atrófica. A forma exsudativa (neovascularizada) evolui com neovascularização de coroide podendo ser classificada como neovascularização clássica, a qual é perfeitamente identificada pelo exame de angioflurosceinografia da retina, e a neovascularização oculta com alguns sinais sugerindo a presença da neovascularização de coróide na angiografia fluresceínica e que, algumas vezes, se identifica o neovaso coroidiano pelo exame de angiografia com indocianina verde ${ }^{(5)}$.

Um terceiro tipo de neovascularização de coróide, sugerido é a proliferação angiomatosa da retina, chamada de "RAP" (6), sendo também chamada de anastomose coroidoretiniana "OCRA" $(7)$.

A forma neovascular da DMRI é caracterizada por um crescimento de vasos sanguíneos no espaço sub-retiniano, denominada membrana neovascular sub-retiniana (MNSR) ou neovascularização de coroide. A MNSR estende-se anteriormente através de um defeito na membrana de Bruch para o espaço abaixo do epitélio pigmentar da retina (EPR), e/ou da retina neurosensorial, ou entre o epitélio pigmentar da retina, levando ao acúmulo de líquido, sangue e até lipídios no espaço sub-retiniano ${ }^{(1)}$.

Tem sido sugerida uma causa genética que poderia explicar o aparecimento da DMRI, que seria o fator de complemento $\mathrm{H}$ e seu polimorfismo. Tal fator e o polimorfismo podem ser responsáveis por quase metade dos 15 milhões de casos de degeneração macular nos $\operatorname{EUA}^{(8,9)}$.

Como muitas doenças crônicas, a DMRI está relacionada a uma combinação de genética e fatores de risco ambientais, que contribuem para o seu aparecimento, como idade, tabagismo, história familiar, obesidade, sedentarismo e hipertensão arterial sistêmica ${ }^{(10)}$.

A DMRI não afeta a visão periférica, não causando perda completa da visão, no entanto pode ocasionar diminuição da visão central súbita pela presença da neovascularização de coróide "RAP" ou vasculopatia polipoidal coroidiana, e achados como: hemorragia retiniana e/ou coroidiana, descolamento da retina sensorial e do epitélio pigmentar da retina. A diminuição da acuidade visual também pode ser lenta pela progressão da degeneração macular seca (não neovascularizada), inicialmente detectada ou não(1).

Quanto à localização da neovascularização de 
coróide esta pode ser subfoveal ou extrafoveal. A forma de localização pode sugerir diferentes modalidades terapêuticas ${ }^{(1)}$.

Atualmente, para a forma subfoveal utilizam-se medicamentos à base de anticorpos monoclonais que atuam no fator específico de crescimento vascular endotelial (VEGF), como o Ranibizumab ${ }^{(11,12)}$ e o Bevacizumab, ambos injetados intravítreo (intraocular) $^{(13,14)}$.

Outra forma de tratamento é um oligonucleotídeo polietilenoglicolado modificado, o qual se liga ao VEGF165 extracelular com alta especificidade e afinidade, inibindo sua atividade que é o Pegaptanibe sódico (Macugen) ${ }^{(15)}$.

Formas combinadas de tratamento que utilizam além do anticorpo monoclonal têm sido usadas em combinação com a terapia fotodinâmica (PDT), dexametasona $^{(16)}$ ou triancinolona intraocular ${ }^{(17)}$. A fototrombose com indocianina verde também foi utili$\operatorname{zada}^{(18)}$.

$\mathrm{Na}$ forma extrafoveal tem sido utilizada a fotocoagulação a laser térmico, com recidiva e dano ao campo visual ${ }^{(15)}$ e a terapia fotodinâmica (PDT) com resultados imprecisos ${ }^{(19)}$. Relatos recentes referem o uso de Ranibizumab e Bevacizumab com bons resultados na forma extrafoveal ${ }^{(20,22)}$.

Desta forma, este trabalho visa avaliar a resposta à injeção intravítrea de Ranibizumab, 0,5mg, em monoterapia, em pacientes portadores de DMRI na sua forma exsudativa com neovascularização de coróide de localização não subfoveal, podendo ser extrafoveal ou justafoveal e fazer uma revisão da literatura.

\section{Овjetivo}

Avaliar a eficácia da injeção intravítrea de Ranibizumab (anti-VEGF) no tratamento da neovascularização de coróide não subfoveal, podendo ser extrafoveal ou justafoveal causada pela degeneração macular relacionada à idade exsudativa e revisão da literatura.

\section{Métodos}

Foi realizado um estudo descritivo e transversal com análise de uma série de casos de 15 pacientes, de ambos os sexos, entre 55 e 78 anos, portadores de degeneração macular relacionada à idade exsudativa com neovascularização de coroide extrafoveal ou justafoveal. Os pacientes foram tratados com uma injeção intravítrea, por mês, no mínimo por três meses consecutivos, de Ranibizumab na dose de $0,5 \mathrm{mg}$, no olho com neovascularização de coróide extrafoveal, seguindo estudo já descrito ${ }^{(23)}$. Estudos ${ }^{(11,12)}$ comprovaram a eficácia e a segurança da droga na dose estabelecida para a pesquisa de $0,5 \mathrm{mg}$.

Aqueles pacientes foram examinados com um mês de intervalo entre as aplicações das injeções e acompanhados com exames complementares que incluíram, além do exame da acuidade visual e do exame oftalmológico de rotina, a angiografia fluoresceínica e tomografia de coerência óptica (TCO).

Foram excluídos da pesquisa casos com neovascularização de coróide subfoveal, inflamatórias, relacionada à miopia, estrias angióides ou degeneração macular relacionada à idade já tratada por algum outro método.

O acompanhamento dos pacientes foi realizado através de protocolo exclusivo criado pelos próprios pesquisadores, no qual foram transcritos os achados de cada exame efetuado nos doentes, facilitando, assim, a descrição dos resultados. Os dados coletados foram utilizados na confecção de tabelas, com intuito de descrever os resultados encontrados.

Foram retratados aqueles pacientes que após três injeções ainda apresentaram piora da melhor visão conseguida com o tratamento ou aumento do fluido sub-retiniano da melhor absorção obtida com o tratamento. Neste caso foi utilizado o método injeta e esten$\mathrm{de}^{(24)}$, mundialmente reconhecido.

\section{Resultados}

Os resultados da análise dos quinze pacientes que foram incluídos nesta série de casos mostraram que oito $(53,3 \%)$ pacientes eram do sexo feminino e sete $(46,6 \%)$ do sexo masculino, e a média de idade encontrada foi de 71,4 anos.

Dos quinze pacientes estudados, onze $(73,3 \%)$ pacientes apresentavam neovascularização de coroide extrafoveal e quatro $(26,6 \%)$ justafoveal. Oito $(53,3 \%)$ apresentavam a forma oculta e sete $(46,6 \%)$ tinham lesões clássicas.

A média de acuidade visual no momento do diagnóstico foi de $0,44 \log$ MAR e a média final de $0,27 \log M A R$.

A média de injeções realizadas foi de 3,67, sendo o menor número de injeções três e o maior seis injeções. Oito $(53,3 \%)$ pacientes mostraram resolução do quadro exsudativo neovascular com o máximo de três injeções 
Tabela 1

Sexo e idades de todos os pacientes do estudo

\begin{tabular}{ccc}
\hline Paciente & Sexo & Idade \\
\hline 1 & Feminino & 65 \\
2 & Masculino & 75 \\
3 & Feminino & 78 \\
4 & Feminino & 73 \\
5 & Masculino & 72 \\
6 & Feminino & 55 \\
7 & Masculino & 76 \\
8 & Masculino & 77 \\
9 & Masculino & 55 \\
10 & Feminino & 78 \\
11 & Feminino & 77 \\
12 & Masculino & 76 \\
13 & Masculino & 75 \\
14 & Feminino & 70 \\
15 & Feminino & 69 \\
\hline
\end{tabular}

Tabela 2

Dados individuais de todos os pacientes do estudo

\begin{tabular}{cccccccccc}
\hline Paciente & $\begin{array}{c}\text { Local da } \\
\text { neovascularização }\end{array}$ & Tipo & $\begin{array}{c}\text { AV } \\
\text { inicial } \\
\text { Snellen }\end{array}$ & $\begin{array}{c}\text { AV } \\
\text { inicial } \\
\text { logMAR* }\end{array}$ & $\begin{array}{c}\text { OCT } \\
\text { (microns) }\end{array}$ & $\begin{array}{c}\mathbf{N}^{\mathbf{2}} \\
\text { Injeções }\end{array}$ & $\begin{array}{c}\text { AV } \\
\text { final } \\
\text { Snellen }\end{array}$ & $\begin{array}{c}\text { AV } \\
\text { final } \\
\text { logMAR* }\end{array}$ & $\begin{array}{c}\text { Ganho } \\
\text { de linhas } \\
\text { de visão }\end{array}$ \\
\hline 1 & Justafoveal & Oculto & $20 / 60$ & 0,47 & 328 & 4 & $20 / 40$ & 0,30 & +2 \\
2 & Justafoveal & Oculto & $20 / 50$ & 0,39 & 286 & 6 & $20 / 30$ & 0,17 & +2 \\
3 & Extrafoveal & Oculto & $20 / 50$ & 0,39 & 280 & 4 & $20 / 40$ & 0,30 & +1 \\
4 & Extrafoveal & Oculto & $20 / 40$ & 0,30 & 270 & 3 & $20 / 30$ & 0,17 & +1 \\
5 & Extrafoveal & Oculto & $20 / 80$ & 0,60 & 260 & 4 & $20 / 50$ & 0,39 & +3 \\
6 & Justafoveal & Clássico & $20 / 60$ & 0,47 & 288 & 4 & $20 / 40$ & 0,30 & +2 \\
7 & Justa-foveal & Clássico & $20 / 80$ & 0,60 & 310 & 3 & $20 / 50$ & 0,39 & +3 \\
8 & Extrafoveal & Oculto & $20 / 60$ & 0,47 & 306 & 3 & $20 / 40$ & 0,30 & +2 \\
9 & Extrafoveal & Clássico & $20 / 50$ & 0,39 & 248 & 3 & $20 / 30$ & 0,17 & +2 \\
10 & Extrafoveal & Clássico & $20 / 50$ & 0,39 & 260 & 5 & $20 / 30$ & 0,17 & +2 \\
11 & Extrafoveal & Clássico & $20 / 50$ & 0,39 & 382 & 4 & $20 / 40$ & 0,30 & +2 \\
12 & Extrafoveal & Clássico & $20 / 50$ & 0,39 & 240 & 3 & $20 / 40$ & 0,30 & +1 \\
13 & Extrafoveal & Oculto & $20 / 40$ & 0,30 & 228 & 3 & $20 / 30$ & 0,17 & +1 \\
14 & Extrafoveal & Clássico & $20 / 80$ & 0,60 & 322 & 3 & $20 / 40$ & 0,30 & +4 \\
15 & Extrafoveal & Oculto & $20 / 60$ & 0,47 & 268 & 3 & $20 / 50$ & 0,39 & +1 \\
\hline
\end{tabular}

* $\log \mathrm{MAR}=$ logaritmo do mínimo ângulo de resolução

e apenas um $(6,67 \%)$ paciente necessitou de seis injeções para resolução do seu quadro.

A acuidade visual máxima alcançada foi de 20/ 30 , e ocorreu em cinco $(33,3 \%)$ pacientes, e a acuidade visual mais baixa alcançada foi de 20/50, ocorrendo em três $(20 \%)$ pacientes.

$\mathrm{Na}$ avaliação final dos pacientes após os seis meses de estudo, a média de linha de visão ganha foi de 1,87 , linha de acuidade visual pela tabela de Snellen, sendo que todos os quinze pacientes ganharam uma ou mais linhas de visão. Seis $(40 \%)$ pacientes conseguiram uma linha de visão, seis (40\%) pacientes ganharam duas linhas de visão, dois $(13,3 \%)$ alcançaram três linhas de visão e um $(6,6 \%)$ ganhou quatro linhas de visão.

\section{Discussão}

Os achados deste estudo respaldam o uso do antiangiogênico na degeneração macular relacionada à idade com neovascularização de coróide extrafoveal clás- 


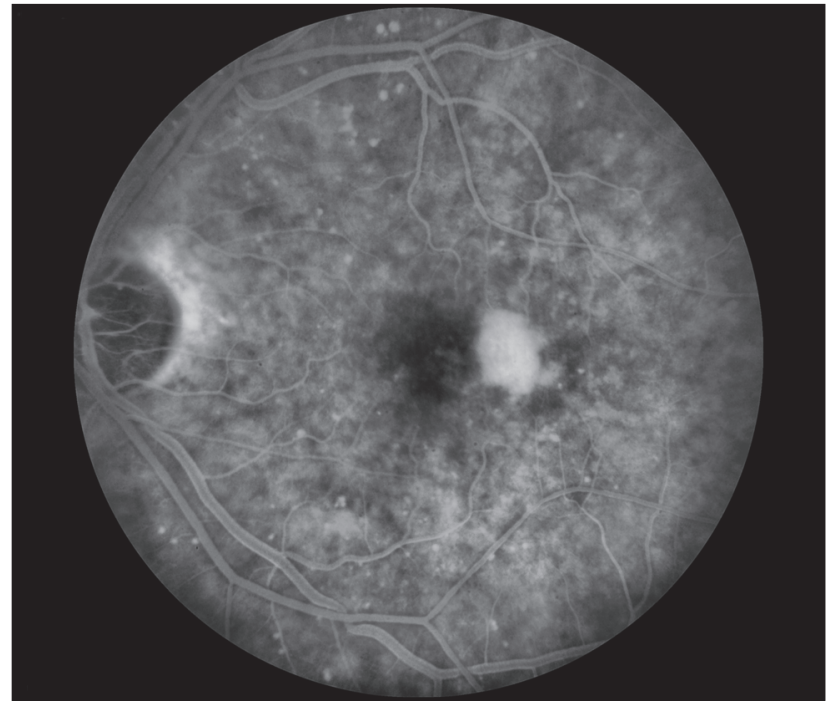

Figura 1: Paciente 3, neovascularização de coróide oculta, extrafoveal

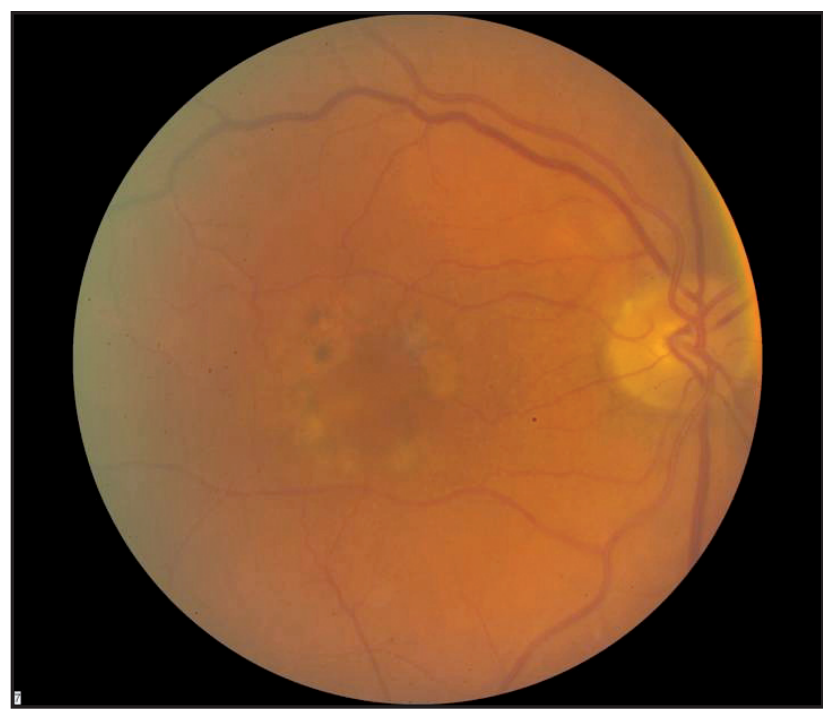

Figura 4: Paciente 10, neovascularização de coróide extrafoveal clássica

sica e oculta, e são similares aos dos estudos multicêntricos ${ }^{(11,12)}$ para degeneração macular relacionada à idade com neovascularização de coróide subfoveal, e também para o tratamento da neovascularização de coróide extrafoveal, como também sugerem outros autores ${ }^{(20-22)}$.

A prevalência da neovascularização de coroide não subfoveal foi avaliada em vários estudos. Em um trabalho $^{(25)}$ retrospectivo com 3.580 casos consecutivos de DMRI neovascular, foram encontrados $18,4 \%$ de lesões justafoveais e $7,7 \%$ de lesões extrafoveais. Similar aos achados de um estudo transversal ${ }^{(26)} \mathrm{com} 16,5 \%$ de lesões justafoveais e $5 \%$ de lesões extrafoveais entre os 200 casos de DMRI neovascular e $47 \%$ das lesões

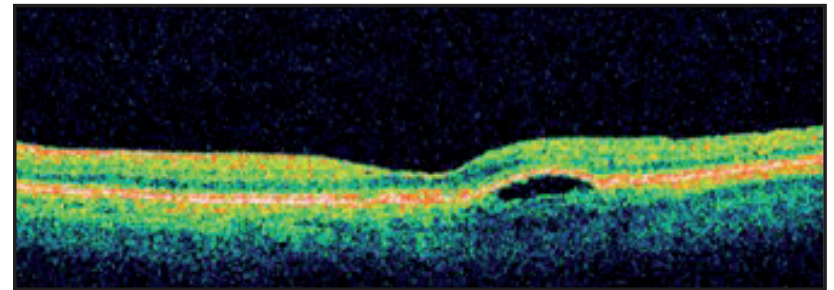

Figura 2: OCT de paciente 3 antes do tratamento com descolamento do epitélio pigmentar da retina

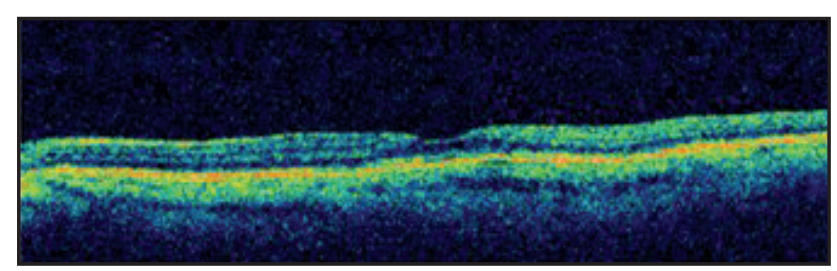

Figura 3: OCT de paciente 3 após o tratamento com resolução do descolamento do epitélio pigmentar da retina

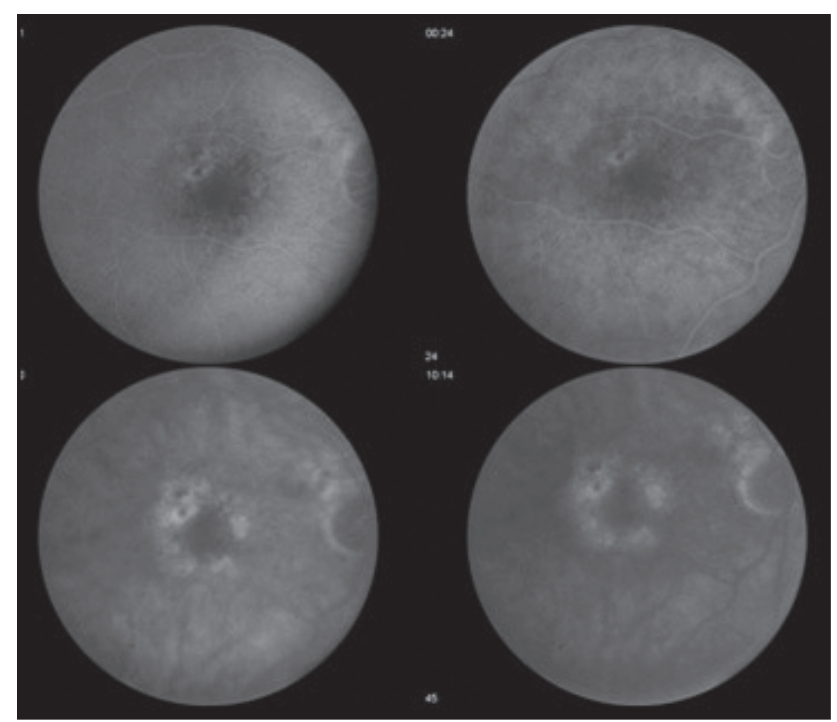

extrafoveal eram predominantemente clássicas. Contrastando com os resultados encontrados na presente pesquisa, na qual houve um predomínio da localização da neovascularização extrafoveal $(73,3 \%)$ sobre a justafoveal $(26,6 \%)$.

Apesar de outras possibilidades terapêuticas já terem sido avaliadas, como a fotocoagulação com laser e a terapia fotodinâmica (PDT), recentes trabalhos e este mostraram que os resultados funcionais são extremamente favoráveis à indicação de antiangiogênico ${ }^{(20,21)}$ (no caso deste estudo o Ranibizumab) comparado à fotocoagulação com laser térmico e ao PDT.

A fotocoagulação com laser tem sido a terapia padrão no manejo da DMRI com neovascularização de 


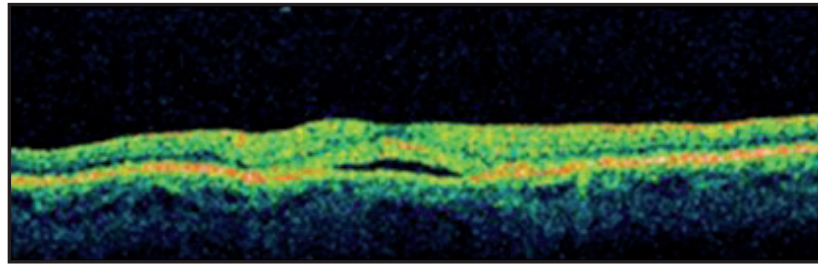

Figura 5: OCT paciente 10 antes do tratamento com fluido entre o epitélio pigmentar da retina

coróide extrafoveal, nas últimas duas décadas. O grupo de estudo de fotocoagulação macular $(1990)^{(27)}$ mostrou que $41 \%$ dos pacientes com lesões extrafoveal e $54 \%$ dos pacientes com lesões justafoveal tinham neovascularização recorrentes após doze meses de seguimento.

Quanto à terapia fotodinâmica com verteporfina, trabalhos relataram resultados variáveis com esta forma de tratamento ${ }^{(19,28)}$. A média das injeções realizadas de 3,67 está de acordo com trabalhos recentes sobre média de injeções em neovascularização de coróide sub-foveal, como sugerido ${ }^{(23)}$, estudo seguido no esquema de tratamento inicial para os casos desta série, complementados pelo método já citado, injeta e estende ${ }^{(24)}$.

O resultado final de ganho de linhas de visão em todos os pacientes estudados, reforça a idéia que em casos de degeneração macular relacionada à idade com neovascularização de coróide extrafoveal, a indicação do Ranibizumab é eficaz e pode ser utilizada como primeira opção para tais casos.

\section{Conclusão}

No tratamento dos pacientes com degeneração macular relacionada à idade e neovascularização de coróide extrafoveal a injeção de Ranibizumab mostrouse eficaz, podendo ser a opção como indicação inicial de tratamento nesses casos.

Agradecimentos: Ao professor James Folk, University of Iowa, pela contribuição na realização deste artigo.

\section{REFERÊNCIAS}

1 Gass JDM. Stereoscopic atlas of macular diseases: diagnosis and treatment. 2nd ed. St. Louis: Mosby; 1977. p.66.

2 Friedman DS, O’Colmain BJ, Muñoz B, Tomany SC, McCarty C, de Jong PT, Nemesure B, Mitchell P, Kempen J; Eye Diseases Prevalence Research Group. Prevalence of age-related macular degeneration in the United States. Arch Ophthalmol. 2004;122(4):564-72.

3 Gass JDM. Pathogenesis of macular detachment and degeneration. Ophthalmic Forum. 1984;2: 8-17.

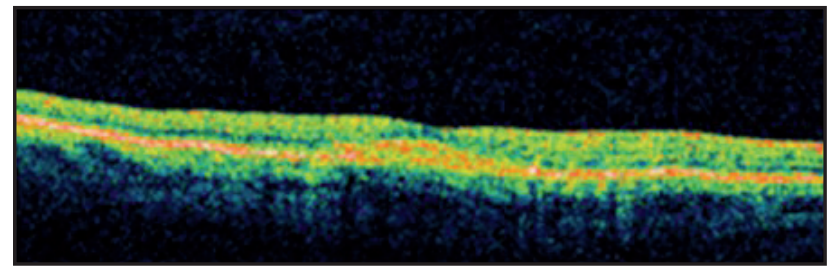

Figura 6: OCT paciente 10 após o tratamento com desaparecimento do fluido entre o epitélio pigmentar da retina

4 Yannuzzi LA, Slakter JS, Sorenson JA, Guyer DR, Orlock DA. Digital indocyanine green videoangiography and choroidal neovascularization. Retina. 1992;12(3):191-223.

5 Freund KB, Ho IV, Barbazetto IA, Koizumi H, Laud K, Ferrara $\mathrm{D}$, et al. Type 3 neovascularization: the expanded spectrum of retinal angiomatous proliferation. Retina. 2008;28(2):201-11.

6 Gass JDM, Agarwal A, Lavina AM, Tawansy KA. Focal inner retinal hemorrhages in patients with drusen: an early sign of occultr choroidal neovascularization and chorioretinal anastomosis. Retina. 2003;23(6):741-51.

7 Klein RJ, Zeiss C, Chew EY, Tsai JY, Sackler RS, Haynes C, et al. Complement factor $\mathrm{H}$ polymorphism in age-related macular degeneration. Science. 2005;308(5720):385-9.

8 Haines JL, Hauser MA, Schmidt S, Scott WK, Oslon LM, Gallins $\mathrm{P}$, et al. Complement factor $\mathrm{H}$ variant increases the risk of age-related macular degeneration. Science.2005;308(5720):419-21.

9 Smith W, Assink J, Klein R, Mitchell P, Klaver CC, Klein BE, et al. Risk factors for age-related macular degeneration: Pooled findings from three continents. Ophthalmology. 2001;108(4):697-704.

10 Brown DM, Kaiser PK, Michels M, Soubrane G, Heier JS, Kim RY, Sy JP, Schneider S; ANCHOR Study Group. Ranibizumab versus verteporfin for neovascular age-related macular degeneration. N Engl J Med. 2006;355(14):1432-44.

11 Rosenfeld PJ, Brown DM, Heier JS, Boyer DS, Kaiser PK, Chung CY, Kim RY; MARINA Study Group. Ranibzumab for neovascular age-related macular degeneration. N Engl J Med. 2006;355(14):1419-31

12 Rich RM, Rosenfeld PJ, Puliafito CA, Dubovy SR, Davis JL, Flynn HW Jr, et al. Short-term safety and efficacy of intravitreal bevacizumab (Avastin) for neovascular age-related macular degeneration. Retina. 2006;26(5):495-511.

13 Rosenfeld PJ, Moshfeghi AA, Puliafito CA. Optical coherence tomography findings after an intravitreal injection of bevacizumab (avastin) for neovascular age-related macular degeneration. Ophthalmic Surg Lasers Imaging. 2005;36(4):331-5

14 Gragoudas ES, Adamis AP, Cunningham ET Jr, Feinsod M, Guyer DR; VEGF Inhibition Study in Ocular Neovascularization Clinical Trial Group. Pegaptanib for neovascular age-related macular degeneration. N Engl J Med. 2004;351(27):2805-16.

15 Argon green vs krypton red laser photocoagulation of extrafoveal choroidal neovascular lesions. One-year results in age-related macular degeneration. The Canadian Ophthalmology Study Group. Arch Ophthalmol. 1993;111(2):181-5.

16 Etter J, Fekrat S. Photodynamic therapy and inravitreal triamcinolone for extrafoveal choroidal neovascularization in neovascular age-related macular degeneration. Ann Ophthalmol. 2006;38(3):239-41. 
17 Augustin AJ, Puls S, Offermann I. Triple therapy for choroidal neovascularization due to age-related macular degeneration: verteporfin PDT, bevacizumab, and dexamethasone. Retina. 2007;27(2):133-40.

18 Cardillo JA, Jorge R, Costa RA, Nunes SM, Lavinsky D, Kuppermann BD, Tedesco AC, Farah ME.Experimental selective choriocapillaris photothrombosis using a modified indocyanine green formulation. Br J Ophthalmol. 2008 Feb;92(2):276-80

19 Spaide RF, Sorenson J, Maranan L. Photodinamic therapy with verteporfin combined with intravitreal injection of triancinolone acetonide for choroidal neovascularization. Ophthalmology. 2005;112(2):301-4.

20 Roller AB, Amaro MH. Intravitreal ranibizumab and bevacizumab for the treatment of nonsubfovel choroidal neovascularization in age-related macular degeneration. Arq Bras Oftalmol. 2009;72(5):677-81.

21 Amaro $\mathrm{MH}$, Roller AB. Intravitreal ranibizumab and bevacizumab for the treatment of nonsubfovel choroidal neovascularization of the age-related macular degeneration. Invest Ophthalmol Vis Sci. 2009;50:E-1884.

22 Arias L, Ruiz-Moreno JM, Gómez-Ulla F, Fernandéz M, Montero J. A 1 year retrospective review of ranibizumab for naïve nonsubfoveal choroidal neovascularization secondary to age-related macular degeneration. Retina. 2009;29(10):1444-9.

23 Lalwani GA, Rosenfeld PJ, Fung AE, Dubovy SR, Michels S, Feuer W, et al. A variable-dosing regimen with intravitreal ranibizumab for neovascular age-related macular degeneration: year 2 of the PrONTO Study. Am J Ophthalmol. 2009;148(1):43-58.e1.
24 Spaide R. Ranibizumab according to need: a treatment for age-related macular degeneration. Am J Ophthalmol. 2007;143(4):679-80.

25 Beaumont PE, Kang HK. Lesion morphology in age-related macular degeneration and its therapeutic significance. Arch Ophthalmol. 2006;124(6):807-12.

26 Olsen TW, Feng X, Kasper TJ, Rath PP, Steuer ER. Fluorescein angiographic lesion type frequency in neovascular agerelated macular degeneration. Ophthalmology. 2004;111(2):250-5.

27 Persistent and recurrent neovascularization after krypton laser photocoagulation for neovascular lesions of age-related macular degeneration. Macular Photocoagulation Study Group. Arch Ophthalmol.1990;108(6):825-31.

28 Blair MP, Apte RS, Miskala PH, Bressler SB, Goldberg MF, Schachat AP, Bressler NM. Retrospective case series of juxtafoveal choroidal neovascularization treated with photodynamic therapy with verteporfin. Retina. 2004;24(4):501-6.

Endereço para Correspondência:

Miguel Hage Amaro

Trav. Quintino Bocaiúva, $\mathbf{n}^{\circ} 516$

CEP 66.053-240 - Belém, (PA), Brasil

Telefone: (91) 3223-6741

E-mail: miguelhamaro@yahoo.com.br 